\title{
Thermal measurements of power semiconductor devices using thermographic system
}

\author{
by B. WIECEK(*), M. GRECKI(*), J.PACHOLIK(*)
}

(") Politechnika Lodzka, Instytut Elektroniki, ul. Stefanowskiego 18/22, 90-924 Lodz, Poland.

\begin{abstract}
The non-uniformity of temperature distribution in the semiconductor structure may cause some problems, especially when the power dissipated in the device is high. In general, there is no easy way to find out the method of evaluating temperature inside the semiconductor wafer. Thermovision gives the possibilities to obtain temperature maps only on the upper surface of the semiconductor device in direct, non-invasive way. In order to find the hot spots inside the silicon, multidimensional modelling is being used. Certain measurements of power bipolar transistor and thyristors either for static and transient states have been performed. For transient state surveys a unique method of shortening the time of the image scanning has been worked out.
\end{abstract}

\section{Conditions of measurements}

Power bipolar transistors as well as Darlington devices in TO-3 metal case has been investigated. After removing the upper side of the case, there is a direct sight of the semiconductor surface. To ensure uniform emissivity over the whole upper surface and to avoid the mirror reflections, the samples have been covered with the thin layer of the black paint with high emissivity factor evaluated at 0.85 .

In order to ensure the same thermal initial conditions the samples has been placed on a special water-cooled radiator. Temperature of the water has been being kept constant at $40^{\circ} \mathrm{C}$. Such a temperature value allows to reduce the thermal noise of the environment.

\section{Steady-state measurements of power bipolar transistors}

The thermal behaviour of the semiconductor structures of bipolar transistors that work with the same high-power excitation, but with the different electric bias conditions has been investigated. The working point of the element has been being changed in the Safe Operating Area (SOA) from low-current, high-voltage to high-current, low-voltage region. The majority the infrared maps has been nearly identical and dependence upon thE conditions of polarisation has not been observed. Only in the case of BU323A, high-voltage, Darlington power transistor, typically being used for automotive ignition and motor control application, the hot spots has been remarked as shown in figure $A^{*}$. The maximum temperature and localisation of the hot spots are a function of collector-emitter voltage $V_{C E}$ and collector current $I_{C}$ ratio.

Transistors have been being tested for $10 \mathrm{~W}$ of dissipated power and following couple of $V_{C E}$ and $I_{C}$ values:

$\begin{array}{llll}V_{C E} & 5 \mathrm{~V} & 200 \mathrm{~V} & 300 \mathrm{~V} \\ I_{C} & 2 \mathrm{~A} & 50 \mathrm{~mA} & 33 \mathrm{~mA}\end{array}$

The temperature maps presented in figure $A$ correspond to the following condition: $V_{C E}=5 \mathrm{~V}$, $I_{C}=2 \mathrm{~A}$ and $V_{C E}=300 \mathrm{~V}, I_{C}=33 \mathrm{~mA}$, respectively. Darlington device consists of two elements:

\footnotetext{
* The colour plates of this article 45 are located on page $X I$ of the colour gathering, at the end of the book
} 


\section{http://dx.doi.org/10.21611/qirt.1992.045}

high-current power and high-voltage control transistors. For both measurement examples mentioned above, the power has been dissipated in quite different areas. In high-current case the main transistor is the source of heat. As a direct consequence of large area, the heat is generated, temperature distribution is nearly flat all over the semiconductor.

The second high-voltage example is much more interesting. In this case, the control transistor has generated the power and heat. The control transistor is symmetrically splitted into two parts placed close to the bottom side of the device (figure $A$ ). The substantial temperature rise has been observed in form of two hot spots where the control transistor is situated as shown in figure 1 . In this case, not proper cooling as well as too high dissipated power may cause the temperature instability and device failure finally.

\section{Transient state surveys}

There in no direct way to use thermovision for surveys of short dynamic thermal processes. It is relatively easily to trace slow-varying temperature changes going on at least several seconds with the help of computer-controlled thermography [2].

A new approach to transient thermal processes investigations in semiconductors assumes that the measurement system contains a power generator fully controlled by computer and synchronised with the radiation sensor shipment in the camera. Power dissipated in the device is generated typically at the beginning of new scanning period, e.g. new image or new line. The computer controls and varies the power pulse parameters and, in particular, its position in time during the image scanning. The principle of this method is presented in figure 2. The power pulse is generated periodically and synchronously with sensor movement in the camera. Every testing period (field or scanning line), the pulse is being slightly shifted in reference to certain moment, when infrared detector passing over a chosen object point. For this point, computer can easily create short-time thermal characteristic using the set of previously stored thermal images [3].

Similarly, assuming that temperature variation during single scan line is negligible, we can completely rebuilt entire image. Using the time reference given by vertical and horizontal synchronisation pulses, computer can trigger the power generator in strictly defined moment. Changing the position of the pulse one can vary the time interval between beginning of the thermal process and the start of temperature measurement determined by a chosen image line. After repeating this process a certain number of times, all lines can be glued in one image corresponding to the required time scale. The scan time shortening is recommended in a variety of semiconductor surveys, e.g. thermal impedance measurements and nondestructive maximum ratings investigations. These measurements have been performed for BU323A transistor excited by square power pulse. Approximately, 50 thermal images of heating process have been captured. The curves of the transient thermal responses for BU323A for two different polarisation conditions are presented in figure 3.

In order to improve maximum ratings we need an insight into the semiconductor while working just near its threshold of destruction [2-3]. Lots of efforts are still being taken to obtain some results analytically, by power device modelling in electrical, thermal, and lately in electrothermal way [3]. Modelling is being carried out under the certain conditions and assumptions, what means the need of practical, measuring verification.

One way to perform such kinds of investigations is to trace the process until the device destruction. The last moment, e.g. second breakdown in transistors when the thermal feedback takes place, is typically very short. It may go on during tens or hundreds of microseconds and, unfortunately this process is not reversible. It makes very difficult to use thermography in such kind of surveys.

The another approach to these investigations is to apply very high power excitation in relatively short time, protecting device from its destruction and making testing process reversible and repetitive. For example, there is no problem to comply periodic current pulse of 
$1000 \mathrm{~A}$ with duration time of hundreds of microseconds to low-current $\left(l_{A V}=40 \mathrm{~A}\right)$ thyristor. In this case, we can easily apply the method of measurement the rapid, short-time thermal processes.

The chosen results of two-dimensional electrothermal modelling are presented in figure 4 We can model either on-state condition with high-power dynamic excitation or temperature effects taking place during plasma spreading in thyristors [2-3]. In order to verify modelling and confirm its correctness, the surveys have been performed for on-state. The thyristor has been initially switched on with the use of low initial current varied over the range of $I_{p}=0.5-2 \mathrm{~A}$. Low initial current flowing in the device just before the high excitation allows to notice the effect of non-uniform current density distribution in on-state, which has never been observed with the use of thermovision.

The temperature under the gate area is lower, what has been confirmed by calculations. It is caused by lower current density as well as lower carriers concentration in this area. The carriers flowing between anode and cathode, need longer way under the gate, where there is no cathode contact.

The temperature on the upper plane as well as inside the device depends on the value of initial forward current. For lower current which flows just before the power impulse, thyristor conducts locally, even in the case of being already in on-state. For low current density and low carrier concentration, the eventual defects in crystal structure or incorrectness in doping profile that involve the changes of recombination surplus, create locally conductive paths in the semiconductor. In effect, during the first hundreds of microseconds of the large electrical shock, the current flows just through already-prepared low-resistance conductive paths. After this time plasma is spreading all over the device. This phenomenon implies the higher local temperature in the semiconductor structure. The temperature characteristics (figure 4) of the point placed at the inner edge of the emitter ring well illustrates the phenomenon of local conductivity of P-N-P-N device in on-state.

\section{Conclusions}

The examples of thermography application presented above well confirm its usefulness, particularly in advanced transient measurements of semiconductor power devices. We have to emphasise that the temperature rise in semiconductor is higher measured by thermography than with the use of traditional electrical methods. It is caused by the fact of neglecting nonuniformity of the temperature distribution all over the semiconductor structure with the use of electric methods.

The thermography measurements with support of 3-D modelling of thermal processes in semiconductor devices are expected to be very good tool for thermal design of power semiconductor devices and their cooling environment.

\section{REFERENCES}

[1] The Probeye Series 4000 Thermal Video Systems. Hughes Aircraft Co.

[2] Lucinski (J.), Napieralski (A.), Wiecek (B.), Grecki (M.), Pacholik (J.). - Methods, algorithms and elements of thermography system for surveys and design power devices and circuits, especially for transient states. Part 1 and 2. Technical University of Lodz, Poland, 1990-1991, work supported by Polish Ministry of National Education.

[3] Wiecek (B.), Grecki (M.). - Two-dimensional modelling of P-N-P-N power devices in on-state and its thermography verification. IASTED Conf. Proc. Modelling, Identification, Control, Innsbruck, Feb. 1991. 
[4] Wiecek (B.). - Electrothermal, two-dimensional analysis of turn-on process in P-N-P-N power devices. Electronics and Telecommunication Warsaw, No.3. 1990.

[5] Wiecek (B.). - Methods and circuits for thermal image conversion. Patents: P-287928, P-276525, Technical University of Lodz, Poland, 1989,1990.

[6] Lucinski (J.), Wiecek (B.), Pacholik (J.). - Power losses for turn-off process in GTO. Conf.

Proc. Circuits Theory and Electronic Circuits, Bielsko-Biala, Oct, 1990,( in polish).

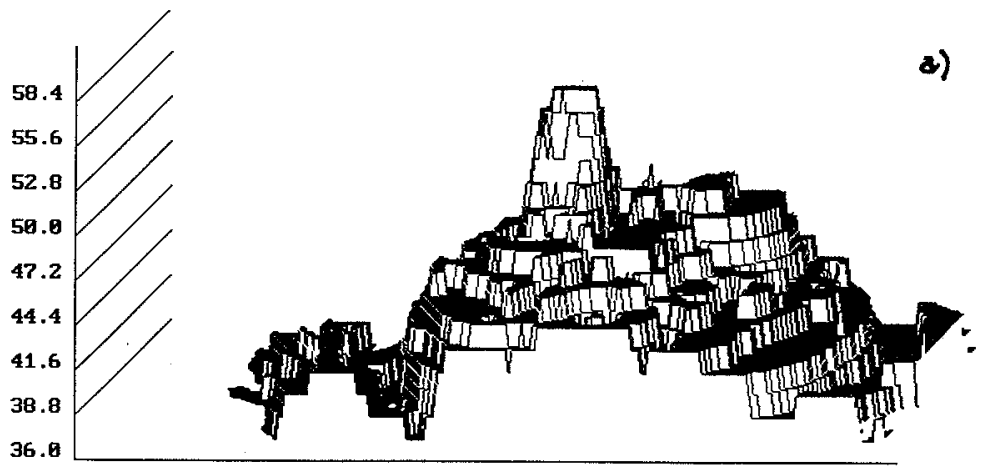

b)

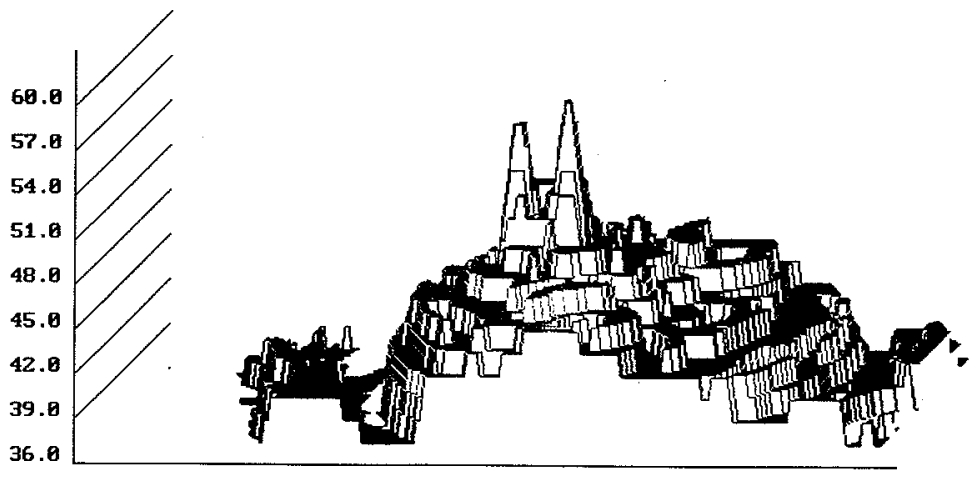

Fig.1. - 3-D shapes of the temperature distribution, BU323A

a) $V_{C E}=5 \mathrm{~V}, I_{C}=2 \mathrm{~A}$, b) $V_{\mathrm{CE}}=300 \mathrm{~V}, I_{\mathrm{C}}=33 \mathrm{~mA}$ 


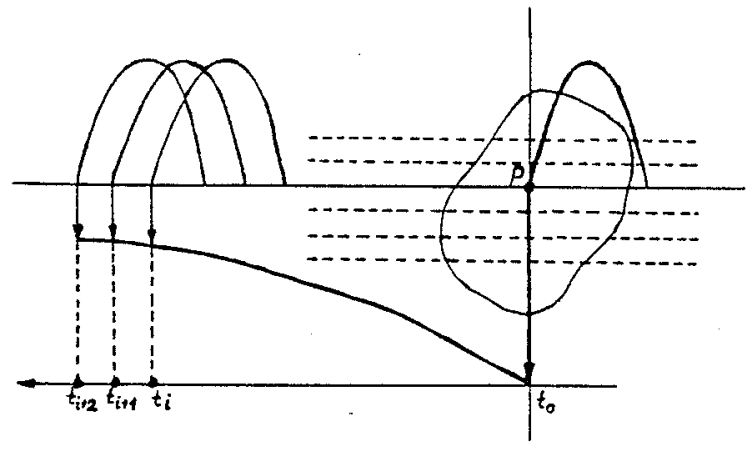

Fig.2. - Principle of short-time, thermal process investigations

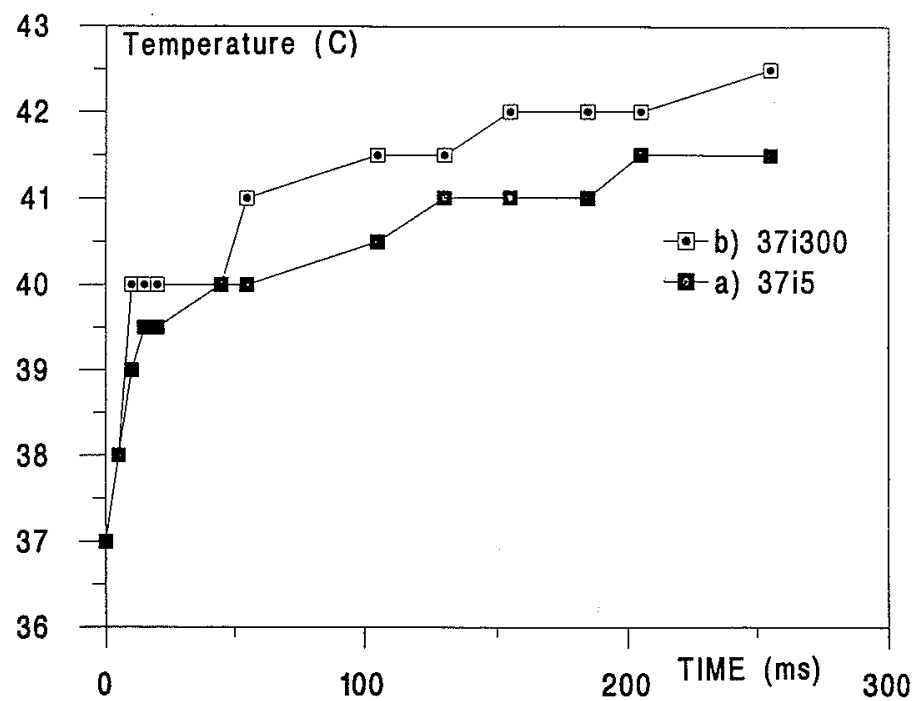

Fig.3. - Thermal response, BU323A

a) $V_{C E}=5 V, I_{C}=2 A$; b) $V_{C E}=300 V, I_{C}=33 \mathrm{~mA}$
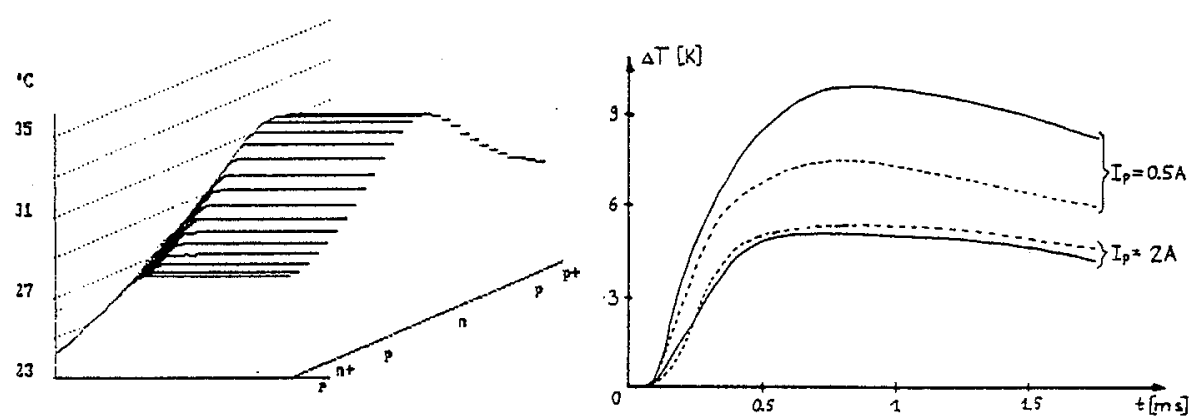

Fig.4. - Temperature inside the semiconductor, modelling and measurement 